\title{
Superspace Parafermions
}

\author{
Constantin Candu and Volker Schomerus \\ DESY Theory Group, DESY Hamburg, Notkestrasse 85, D-22603 Hamburg, Germany
}

(Dated: April 2011)

\begin{abstract}
We describe several families of non-unitary coset conformal field theories that possess truly marginal couplings. These generalize the known examples of Wess-Zumino-Witten models on supergroups such as PSU $(n \mid n)$ or $\operatorname{OSP}(2 n+2 \mid 2 n)$. Our extension includes coset space sigma models, affine Toda theories or Gross-Neveu models which are believed to arise in certain limits.

PACS numbers: 11.25. HF; 11.25.-w; 11.25. Sq.
\end{abstract}

\section{INTRODUCTION}

Non-unitary 2-dimensional (2d) field theories provide a rich and important class of models. They describe surface statistical mechanics systems and they underly the construction of exact string backgrounds. It is very difficult to obtain any systematic statements about these theories since many of the established methods heavily rely on unitarity. Hence very little is known e.g. about the classification of renormalization group fixed points.

Within the class of non-unitary $2 \mathrm{~d}$ field theories, there is a special subset of models that possess some (hidden) internal supersymmetry, i.e. models that admit the action of some (deformed) superalgebra in field space. Intuition suggests that supersymmetry makes these models more accessible. On the other hand, such supersymmetric $2 \mathrm{~d}$ field theories still possess highly relevant applications e.g. to the study of disordered systems [1] or strings in Anti-deSitter backgrounds [2].

The models we are about to study can be considered as a vast extension of the $Z_{N}$ parafermions discovered by Fateev and Zamolodchikov in [3]. These provide a family of $2 \mathrm{~d}$ conformal field theories with central charges $c=2-6 /(k+2)$ whose first members are the well known Ising $(k=2)$ and three-states Potts model $(k=3)$. A few years later, Fateev argued that $Z_{N}$ parafermions possess an integrable perturbation by a relevant field of dimension $h=(k-1) / k[4]$. This perturbation was studied further in [5] and the limit $k \rightarrow \infty$ was identified with the the o(3) sigma model [6, 7]. $Z_{N}$ parafermions were subsequently shown to arise as special cases of the socalled GKO coset construction of Goddard, Kent and Olive [8, 9]. In fact, $Z_{N}$ parafermions emerge from the GKO coset model sU(2)/U(1). Many of the features that were initially found for $Z_{N}$ parafermions could be extended to more general cosets. The potential relation between perturbed GKO cosets and conventional coset sigma models has also been investigated [10, 11].

Here we propose to consider several families of superspace GKO coset models along with their perturbations. The simplest representatives are the cosets of the form $\operatorname{PSU}(2 \mid 2) / \mathrm{SU}(1 \mid 2)$. These provide a family of non-unitary conformal field theories with central charge $c=-2$ which is independent of the level. In contrast to their bosonic relatives, the $\mathrm{PSU}(2 \mid 2) / \mathrm{SU}(1 \mid 2)$ cosets turn out to possess a perturbation of dimension $h=1$. We shall show that the corresponding $\beta$-function vanishes at two loops.

This result can be generalized to other GKO coset models involving supergroups as follows. Introduce a Lie supergroup $\mathrm{G}$ along with a subgroup $\mathrm{H}$. We shall denote the corresponding Lie superalgebras by $\mathfrak{g}$ and $\mathfrak{h}$, respectively. The linear space $\mathfrak{m}=\mathfrak{g} / \mathfrak{h}$ carries a representation of $\mathfrak{h}$. We assume that both $\mathfrak{g}$ and $\mathfrak{h}$ are semisimple. Decomposing $\mathfrak{h}=\oplus_{\nu=1}^{p} \mathfrak{h}_{\nu}$ into simple superalgebras and $\mathfrak{m}=\oplus_{\sigma=1}^{q} \mathfrak{m}_{\sigma}$ into $\mathfrak{h}$-irreducible summands we are able to formulate the main claim.

Proposition: Suppose the quadratic Casimir elements $C_{\nu}^{(2)}$ of $\mathfrak{h}_{\nu}$ on the representation spaces $\mathfrak{m}_{\sigma}$ vanish, i.e.

$$
C_{\nu}^{(2)}\left(\mathfrak{m}_{\sigma}\right)=0
$$

for all $\nu=1, \ldots, p$ and $\sigma=1, \ldots, q$. Then the $G K O$ coset model $\mathrm{G} / \mathrm{H}$ possesses truly marginal deformations for all values of the level.

It is easy to check that the GKO coset PSU $(2 \mid 2) / \mathrm{SU}(1 \mid 2)$ satisfies the condition formulated in our main claim. In this case, $\operatorname{psu}(2 \mid 2) / \mathrm{su}(1 \mid 2)$ is a direct sum of the fundamental representation of $\operatorname{su}(1 \mid 2)$ and its dual. Both have vanishing Casimirs. Our result generalizes previous findings [12] for Wess-Zumino-Witten (WZW) models on supergroups with vanishing dual Coxeter number.

In sec. 2 we describe four families of coset models satisfying the conditions of our main claim, see cases (6 9). We construct explicitly the marginal operators. The corresponding $\beta$-functions are studied in sec. 3 where they are shown to vanish at two loops. Sec. 4 concludes with an overview of applications. In particular, we discuss exactly marginal deformations connecting various GKO cosets. Large level limits shed new light on strong-weak coupling dualities between conformal sigma models and Toda-models for algebras $\operatorname{osp}(2 n+2 \mid 2 n)$ and $\operatorname{psu}(n \mid n)$.

\section{COSETS AND PERTURBATIONS}

We shall denote the WZW model on a supergroup G by $\hat{\mathrm{G}}$. The GKO construction identifies the commutant of the current algebra of $\hat{\mathrm{H}}$ within the field space of $\hat{\mathrm{G}}$ with the chiral algebra of a conformal field theory (CFT) known as the G/H GKO coset or, simply, $\hat{\mathrm{G}} / \hat{\mathrm{H}}$. Given 
some choice for the level(s) of $\hat{\mathrm{G}}$, the levels $k_{\nu}$ of the simple factors $\hat{\mathrm{H}}_{\nu}$ in the denominator are determined through the so-called embedding index of $\mathrm{H}$ into $\mathrm{G}$, see 13 .

Let us show that the $\hat{\mathrm{G}} / \hat{\mathrm{H}}$ model contains fields of weight $(h, \bar{h})=(1,1)$. These marginal fields are of the type $\left(0, \mathfrak{m}_{\sigma}\right)$ in standard GKO coset notations. In order to construct them we introduce a basis $\left\{j_{a}\right\}_{a=1}^{\operatorname{dim} \mathfrak{g}}$ of $\mathfrak{g}$ which respects the grading of $\mathfrak{g}$ and the decomposition

$$
\left.\mathfrak{g}\right|_{\mathfrak{h}}=\bigoplus_{\nu=1}^{p} \mathfrak{h}_{\nu} \oplus \bigoplus_{\sigma=1}^{q} \mathfrak{m}_{\sigma}
$$

of $\mathfrak{g}$ into irreducible representations of $\mathfrak{h}$. This means that every basis vector $j_{a}$ is either bosonic or fermionic, that is has degree $|a|=0$ or $|a|=1$ respectively, and belongs to some summand in eq. (2). For clarity, we shall sometimes attach superscripts G, H and G/H to the fields of the $\hat{\mathrm{G}}$, $\hat{\mathrm{H}}$ and $\hat{\mathrm{G}} / \hat{\mathrm{H}}$ models.

The marginal fields $\left(0, \mathfrak{m}_{\sigma}\right)$ are obtained by decomposing the chiral currents $J_{a}^{\mathrm{G}}$ indexed by $j_{a} \in \mathfrak{m}_{\sigma}$ into a product of fields of the $\hat{\mathrm{H}}$ model and of the $\hat{\mathrm{G}} / \hat{\mathrm{H}}$ model,

$$
J_{a}^{\mathrm{G}}(z)=\psi_{\sigma}^{\mathrm{G} / \mathrm{H}}(z) \otimes \phi_{a}^{\mathrm{H}}(z), j_{a} \in \mathfrak{m}_{\sigma} .
$$

Since the operator product expansion (OPE) between currents $\left\{J_{k}(w)\right\}_{j_{k} \in \mathfrak{h}}$ and $\left\{J_{a}(z)\right\}_{j_{a} \in \mathfrak{m}_{\sigma}}$ only contains simple poles, the same holds for the fields $\phi_{a}$ on the right hand side. Equivalently, the fields $\left\{\phi_{a}\right\}_{j_{a} \in \mathfrak{m}_{\sigma}}$ are primary fields of $\hat{\mathrm{H}}$ within a single affine conformal block. The parafermion fields $\left\{\psi_{\sigma}\right\}_{\sigma=1}^{q}$ are primaries of the $\hat{\mathrm{G}} / \hat{\mathrm{H}}$ model. Equating the conformal weights on both sides of the decomposition formula (3) we conclude that the fields $\psi_{\sigma}$ are indeed marginal

$$
h\left(0, \mathfrak{m}_{\sigma}\right)=1-\sum_{\nu} \frac{C_{\nu}^{(2)}\left(\mathfrak{m}_{\sigma}\right)}{k_{\nu}+c_{\nu}^{\vee}}=1,
$$

where $c_{\nu}^{\vee}$ is the dual Coxeter number of $\mathfrak{h}_{\nu}$. In the second step we have inserted the key assumption (1) of our proposition. We can now deform the $\hat{\mathrm{G}} / \hat{\mathrm{H}}$ model by introducing a marginal perturbation

$$
\mathcal{O}(z, \bar{z})=\sum_{\sigma} \lambda^{\sigma} \mathcal{O}_{\sigma}(z \bar{z})=\sum_{\sigma} \lambda^{\sigma} \psi_{\sigma}(z) \bar{\psi}_{\bar{\sigma}}(\bar{z}),
$$

with couplings $\lambda_{\sigma}$. The fields $\bar{\psi}_{\bar{\sigma}}(\bar{z})$ are constructed similarly from the anti-chiral currents $\bar{J}_{a}(\bar{z})$, but for $j_{a} \in \mathfrak{m}_{\sigma}^{*}$.

Examples: Suppose that $\mathrm{H}$ is the fixed point set of some involution of $\mathrm{G}$, that is the pair $(\mathrm{G}, \mathrm{H})$ corresponds to a symmetric superspace. Then there are four families of coset models that satisfy the conditions in 1, see also 14]. The central charge is either $c=1$ or $c=-2$ depending on wether $\mathrm{G}$ is of OSP or respectively PSU type.

1. Coset theories of real super-Grassmannian type

$$
\frac{\operatorname{OsP}(2 n+2 m+2 \mid 2 n+2 m)_{k}}{\operatorname{OSP}(2 n+1 \mid 2 n)_{k} \times \operatorname{OSP}(2 m+1 \mid 2 m)_{k}} .
$$

In this case, $\mathfrak{m}$ is a bifundamental representation

$$
\mathfrak{m}=\square_{2 m}^{2 m+1} \times \square_{2 n}^{2 n+1},
$$

where $\emptyset_{2 n}^{m}$ denotes the $\operatorname{osp}(m \mid 2 n)$ fundamental representation with Casimir $m-2 n-1$. Since $\mathfrak{m}$ is irreducible, there is one marginal parafermion $\psi$. These are superspace generalizations of the Grassmanian parafermions [5, 10, 11].

2. Coset theories of complex super-Grassmannian type

$$
\frac{\operatorname{PSU}(n+m \mid n+m)_{k}}{\mathrm{SU}(n-1 \mid n)_{k} \times \mathrm{SU}(m+1 \mid m)_{k}} .
$$

For such coset models, $\mathfrak{m}$ is only real irreducible

$$
\mathfrak{m}=\square_{n}^{n-1} \times \overline{\mathbf{u}}_{m}^{m+1} \oplus \overline{\mathbf{u}}_{n}^{n-1} \times \underline{u}_{m}^{m+1}
$$

where $\square_{n}^{m}$ denotes the fundamental representation of $\mathrm{SU}(m \mid n) \cong \mathrm{SU}(n \mid m)$ and its dual carries an additional bar. In both representations, the Casimir assumes the value $m-n-1 /(m-n)$. There are two marginal parafermions $\psi_{+}$and $\psi_{-}$related by the conjugation symmetry $\psi_{+}^{\dagger}=\psi_{-}$inherited from the WZW models. The perturbation (5) respects this symmetry, provided we impose $\lambda_{+}^{*}=\lambda_{-}$on the complex couplings $\lambda_{ \pm}$. This family is a superspace generalization of the usual $\mathrm{SU}(2) / \mathrm{U}(1)$ parafermions. The potential relationship between the $k \rightarrow \infty$ limit of this type of perturbed coset CFTs and the complex Grassmaniann $\sigma$-models with $\theta$-term was suggested in [5].

3. Our third series consists of coset models of the form

$$
\frac{\operatorname{PSU}(2 n \mid 2 n)_{k}}{\operatorname{OSP}(2 n \mid 2 n)_{2 k}} \text {. }
$$

The representation $\mathfrak{m}$ is irreducible for $n>1$

$$
\mathfrak{m}=O O_{2 n}^{2 n}
$$

and is obtained from the osp $(2 n \mid 2 n)$ traceless symmetric tensors of rank 2 by factoring out a 1-dimensional submodule. The reducible but indecomposable traceless tensor of rank 2 has a vanishing Casimir eigenvalue, because its restriction to the 1-dimensional submodule vanishes. For $n=1$ we get the particular case of the series (7) with $m=n=1$ since $\operatorname{OSP}(2 \mid 2)_{2 k} \simeq \mathrm{SU}(2 \mid 1)_{k}$.

4. Diagonal cosets with $\mathrm{G}=\mathrm{H} \times \mathrm{H}$ and $\mathrm{H}$ simple, i.e.

$$
\frac{\mathrm{H}_{k} \times \mathrm{H}_{l}}{\mathrm{H}_{k+l}} .
$$

Since $\mathfrak{m} \simeq \mathfrak{h}$ in this case, assumption 1 requires the adjoint representation of $\mathfrak{h}$ to have a vanishing Casimir. This condition constrains the supergroup H to be $\operatorname{PSU}(n \mid n), \operatorname{OSP}(2 n+2 \mid 2 n)$ or $\mathrm{D}(2,1 ; \alpha)$.

For the cosets (6] 9) one can check that the operator algebra of the parafermions $\psi_{\sigma}$ cannot generate other nontrivial fields of dimension $h \leq 1$, provided the level of $\hat{\mathrm{G}}$ is large enough. This makes the perturbation (5) renormalizable. Notice that $\mathfrak{h}$-invariant level one descendents of $\hat{G}$ primaries with vanishing conformal weight also lead to marginal fields in the $\hat{\mathrm{G}} / \hat{\mathrm{H}}$ theory. Of particular interest 
are the $q$ fields $\chi_{\sigma}=(\mathfrak{g}, 0)_{\sigma}$ in the cosets (6] and the fields $\chi_{1}=(\mathfrak{h} \times 0,0), \chi_{2}=(0 \times \mathfrak{h}, 0)$ for the cosets (9). These "dual" fields are local with respect to $\psi_{\sigma}$ and generate conserved currents [15] in the $\hat{\mathrm{G}} / \hat{\mathrm{H}}$ model perturbed by (5). Local conserved currents of the type $(0,0)$ and of higher spin can also be constructed [16]. These structures should be crucial to argue that the perturbation (5) is integrable. As in the case of the fields $\psi_{\sigma}$ one may show that the operator algebra of the fields $\left\{\chi_{\sigma}\right\}_{\sigma=1}^{q}$ of (6] 8) and of the fields $\chi_{1}$ or $\chi_{2}$ of (9) does not generate other non-trivial fields of dimension $h \leq 1$. This suggests considering the dual perturbation built out of $\chi$ 's instead of $\psi$ 's on the same footing with (5). We differ the analysis of such dual perturbations to our forthcoming work.

\section{VANISHING OF BETA FUNCTION}

The 1-loop $\beta$-function $\beta^{(1)}$ of the CFTs (6 9) perturbed by the operator (5) is given by the coefficient $C_{\rho \sigma}{ }^{\tau}$ that multiplies the operator $\mathcal{O}_{\tau}$ in the operator product expansion (OPE) of the product $\mathcal{O}_{\rho} \mathcal{O}_{\sigma}$,

$$
\beta^{\tau}=\left(1-h\left(0, \mathfrak{m}_{\tau}\right)\right) \lambda^{\tau}-\frac{1}{2} C_{\rho \sigma}{ }^{\tau} \lambda^{\rho} \lambda^{\sigma}+\ldots,
$$

see e.g. 17]. The first term is zero due to eq. (41).

To see that the second term vanishes as well, we adopt the BRST construction of the coset theory in which the coset fields are represented as cohomology classes in the direct product $\hat{\mathrm{G}} \times \hat{\mathrm{H}}$ of WZW models [18]. In the product theory, our marginal parafermions become

$$
\psi_{\sigma}(z)=\sum_{\substack{j_{a} \in \mathfrak{m}_{\sigma} \\ j_{b} \in \mathfrak{m}_{\sigma}^{*}}} J_{a}^{\mathrm{G}}(z) \otimes \phi_{b}^{\mathrm{H}}(z) g^{a b} .
$$

Here we used an invariant non-degenerate form $($,$) of \mathfrak{g}$ to define $g^{a b}$ as the inverse of the matrix $g_{a b}=\left(j_{a}, j_{b}\right)$. The OPE of the fields $\psi_{\sigma}$ involves the structure constants $f_{a b}^{c}$ of $\mathfrak{g}$ defined as usual $\left[j_{a}, j_{b}\right]=f_{a b}^{c} j_{c}$. For symmetric spaces $[\mathfrak{m}, \mathfrak{m}] \subset \mathfrak{h}$. Hence, $f_{a b}^{c}$ vanish if $j_{a}, j_{b}, j_{c} \in \mathfrak{m}$. This proves that $\beta^{(1)}=0$.

The structure of the 4-point function determines whether scale invariance is broken at 2-loops. According to Cardy [19], the 2-loop $\beta$-function $\beta^{(2)}$ vanishes if $\beta^{(1)}=0$ and only the conformal block of the identity contributes to the 4-point function $\left\langle\mathcal{O}_{\kappa} \mathcal{O}_{\rho} \mathcal{O}_{\sigma} \mathcal{O}_{\tau}\right\rangle$. We claim that the 4-point functions in the coset models 69) satisfy such a criterion. In the case of unitary CFTs, Cardy's condition implies that $\psi_{\sigma}$ are abelian currents and exact marginality follows at all loops. This conclusion does not extend to our non-unitary models. Nevertheless, we demonstrate that $\beta^{(2)}=0$ by analyzing the 4-point functions of the perturbing fields.

For simplicity, we restrict our analysis to the supersphere coset models (6). In order to evaluate the 4-point function of the the perturbing field (11) we note that

$$
\begin{aligned}
& \left\langle J_{a}\left|J_{b}(1) J_{c}(z)\right| J_{d}\right\rangle^{\mathrm{G}}=g_{a b} g_{c d} z^{-2}+(-1)^{|b \| c|} g_{a c} g_{b d}+(12) \\
& g_{a d} g_{b c}(1-z)^{-2}+f_{a b}{ }^{m} f_{m c d} z^{-1}+f_{b c}{ }^{m} f_{a m d}(1-z)^{-1}
\end{aligned}
$$

where we have absorbed the levels into $g_{a b}$ and denoted $f_{a b c}=\left(\left[j_{a}, j_{b}\right], j_{c}\right)$. The 4 -point function of the primary fields $\phi^{\mathrm{H}}$ depends on the cross-ratio $z$ through

$$
\begin{gathered}
\left\langle\phi_{a}\left|\phi_{b}(1) \phi_{c}(z)\right| \phi_{d}\right\rangle^{\mathrm{H}}=g_{a b} g_{c d} F_{1}(z)+ \\
(-1)^{|b \| c|} g_{a c} g_{b d} F_{2}(z)+g_{a d} g_{b c} F_{3}(z) .
\end{gathered}
$$

These terms correspond to the three invariants in the tensor product $\mathfrak{m}^{\otimes 4}$. One may determine the (logarithmic) conformal blocks $F_{i}(z)$ with the help of the KnizhnikZamolodchikov (KZ) equation [13]. It implies that

$$
F_{1}(z)+F_{2}(z)+F_{3}(z)=1 .
$$

Contraction of our eqs. (12) and (13) along with eq. (11) eliminates the terms involving $f$, i.e. the last two terms in eq. (12). Then using (14) we obtain

$$
\langle\mathcal{O}|\mathcal{O}(1,1) \mathcal{O}(z, \bar{z})| \mathcal{O}\rangle=\left|1+z^{-2}+(1-z)^{-2}\right|^{2} .
$$

The answer coincides with the 4-point function of the field $J \bar{J}$, where $J$ is an abelian $\mathrm{U}(1)$ current. Therefore, by Cardy's criterion $\beta^{(2)}=0$.

The calculation of $\left\langle\mathcal{O}_{\kappa} \mathcal{O}_{\rho} \mathcal{O}_{\sigma} \mathcal{O}_{\tau}\right\rangle$ for the other cosets (6-9) uses a key fact that follows from eq. (11): There is a unique $\mathfrak{h}$-invariant form $p$ on the space of invariants in $\mathfrak{m}^{\otimes 4}$. Let $F_{J}(z)$ and $F_{\phi}(z)$ denote the value of $p$ on the 4-point function of currents and primaries, respectively. With appropriate normalizations we find

$$
\left\langle\psi_{\kappa}\left|\psi_{\rho}(1) \psi_{\sigma}(z)\right| \psi_{\tau}\right\rangle=F_{J}(z) F_{\phi}(z) .
$$

Application of the $\mathrm{KZ}$ equation yields $F_{\phi}(z)=1$. Furthermore, the last two terms in eq. (12) can be shown to belong to the kernel of $p$. Hence, in calculating the 4-point function, we may treat $\psi_{\sigma}$ as an abelian current. A detailed proof will be presented in the follow-up paper.

\section{APPLICATIONS AND OUTLOOK}

The classification (6) 9) of GKO cosets with exactly marginal couplings has actually appeared first in a seemingly different context, namely in the analysis of $\beta$ functions for sigma models on symmetric superspaces [14] that extends and corrects earlier claims in [20]. This is no accident. It has long been suspected [5, 11] that perturbed $\mathrm{G} / \mathrm{H}$ GKO coset models tend to the associated sigma model on the symmetric space $\mathrm{G} / \mathrm{H}$ in the limit of large levels. Our findings are in perfect agreement with such a correspondence. In fact, we have shown that the 2-loop $\beta$-function of the perturbed $\mathrm{G} / \mathrm{H}$ GKO cosets vanishes if and only if the sigma model on the symmetric superspace $\mathrm{G} / \mathrm{H}$ is conformal. For the sigma models, the vanishing of the $\beta$-function was established non-perturbatively. This suggests that the same should hold for our perturbed GKO coset models, a fact we shall prove directly in our forthcoming paper.

Diagonal cosets $\mathrm{H}_{k} \times \mathrm{H}_{l} / \mathrm{H}_{k+l}$ provide the most important family of GKO coset models. In fact, these theories 
possess many relations with other interesting $2 \mathrm{~d}$ systems. If we set $l=1$ and let $k \rightarrow \infty$ we obtain affine Toda theories for the Lie (super-)algebra $\mathfrak{h}$. Setting $l=2$ instead leads to models with world-sheet supersymmetry. Increasing $l$ further gives affine Toda theories coupled to parafermions [15, 21]. We can also consider a limit in which both $k, l \rightarrow \infty$ while keeping $k-l$ fixed. Thereby we obtain the principal chiral model on the (super-)group $\mathrm{H}$ with a WZ term at level $k-l$. The latter was shown to be conformal in [22, 23]. For a review of various level limits in diagonal cosets see 24].

The relations outlined in the last two paragraphs show that all previously known examples of superspace models with marginal deformations can be recovered from our results by taking appropriate limits. Our findings extend earlier studies and provide a unifying view that suggests interesting new connections between known models.

There is much room for dualities between the perturbed GKO cosets (6 9), i.e. it might be possible that some of our coset models can be deformed into each other by the exactly marginal perturbations we have described. Such deformations should be considered as superspace counterparts of the known massless renormalization group flows between bosonic GKO cosets. Most famous [25] are the level reducing flows of diagonal cosets

\begin{tabular}{|c|c|c|c|c|}
\hline $\mathrm{H}_{k} \times \mathrm{H}_{l}$ & $U V$ & scale & $I R$ & $\mathrm{H}_{k-l} \times \mathrm{H}_{2}$ \\
\hline$\overline{\mathrm{H}_{k+l}}$ & & bation & $\chi_{1}$ & $\mathrm{H}_{k}$ \\
\hline
\end{tabular}

for $k>l$. It seems likely that these are replaced by continuous deformations of CFTs for the diagonal cosets (9). Flows between non-diagonal cosets have been investigated e.g. by Fendley [11]. Following this work, we conjecture that our marginal deformations relate the coset models

$$
\begin{aligned}
\frac{\operatorname{OSP}(2 n+2 \mid 2 n)_{k}}{\operatorname{OSP}(2 n+1 \mid 2 n)_{k}} & \leftrightarrow \frac{\operatorname{OSP}(2 n+2 \mid 2 n)_{k-1} \times \operatorname{OSP}(2 n+2 \mid 2 n)_{1}}{\operatorname{OSP}(2 n+2 \mid 2 n)_{k}} \\
\frac{\operatorname{PSU}(2 n \mid 2 n)_{k}}{\operatorname{OSP}(2 n \mid 2 n)_{2 k}} & \leftrightarrow \frac{\operatorname{PSU}(2 n \mid 2 n)_{k-1} \times \operatorname{PSU}(2 n \mid 2 n)_{1}}{\operatorname{PSU}(2 n \mid 2 n)_{k}} .
\end{aligned}
$$

In the limit $k \rightarrow \infty$, the perturbed coset models on the right hand side tend to the $\operatorname{OsP}(2 n+2 \mid 2 n)$ and PSU $(2 n \mid 2 n)$ Gross-Neveu models, respectively. Both theories are known to be conformal. In the same limit, the first model on the left hand side approaches the conformal sigma model on the supersphere $\operatorname{OsP}(2 n+2 \mid 2 n) / \operatorname{OsP}(2 n+1 \mid 2 n)$, that is a superspace with bosonic base the sphere $S^{2 n+1}$. The duality between the supersphere sigma model and the $\operatorname{OsP}(2 n+2 \mid 2 n)$ Gross-Neveu model was analyzed in much detail before $[26-28]$. In the second relation, the left hand side tends to the sigma model on the coset space $\operatorname{PSU}(2 N \mid 2 N) / \operatorname{OSP}(2 N \mid 2 N)$ which we conjecture to be dual to the $\operatorname{PSU}(2 N \mid 2 N)$ Gross-Neveu model. For the special case $\operatorname{PSU}(2 \mid 2) / \operatorname{OSP}(2 \mid 2) \simeq \mathbb{C} P^{1 \mid 2}$, the duality with the PSU $(2 \mid 2)$ Gross-Neveu model was also conjectured before [29] but no direct evidence was found in [30]. Given our new findings, it seems worthwhile revisiting the issue.

Acknowledgements: We would like to thank Stefan Fredenhagen, Vladimir Mitev, Hubert Saleur and Arkady Tseytlin for very useful discussions and comments. VS is grateful to the Mathematical Physics Group of King's College, London, for their warm hospitality and to the London Mathematical Society for financial support. CC was supported by SFB676.
[1] K. B. Efetov, Adv. Phys. 32 (1983) 53.

[2] A. M. Polyakov, arXiv:hep-th/0512310

[3] V. A. Fateev and A. B. Zamolodchikov, Sov. Phys. JETP 62 (1985) 215 [Zh. Eksp. Teor. Fiz. 89 (1985) 380].

[4] V. A. Fateev, Int. J. Mod. Phys. A6 (1991) 2109.

[5] V. A. Fateev and A. B. Zamolodchikov, Phys. Lett. B271 (1991) 91.

[6] A. B. Zamoldochikov and Al. B. Zamolodchikov, Ann. Phys. 120 (1979) 253.

[7] A. B. Zamoldochikov and Al. B. Zamolodchikov, Nucl. Phys. B379 (1992) 602.

[8] P. Goddard, A. Kent and D. I. Olive, Commun. Math. Phys. 103 (1986) 105.

[9] P. Goddard, A. Kent and D. I. Olive, Phys. Lett. B152 (1985) 88.

[10] P. Fendley, Phys. Rev. Lett. 83 (1999) 4468.

[11] P. Fendley, JHEP 0105 (2001) 050.

[12] T. Quella, V. Schomerus and T. Creutzig, JHEP 0810 (2008) 024 arXiv:0712.3549 [hep-th]].

[13] P. Di Francesco, P. Mathieu and D. Sénéchal, Conformal Field Theory, Springer 1997.

[14] C. Candu, PhD thesis Université Paris 6 (2008) 65-75 [tel.archives-ouvertes.fr/tel-00494973] (in french).

[15] C. Ahn, D. Bernard and A. LeClair, Nucl. Phys. B346
(1990) 409.

[16] T. Eguchi and S. K. Yang, Phys. Lett. B224 (1989) 373.

[17] A. B. Zamolodchikov, JETP Lett. 43 (1986) 730.

[18] D. Karabali and H. J. Schnitzer, Nucl. Phys. B329 (1990) 649.

[19] J. L. Cardy, J. Phys. A20 (1987) L891.

[20] A. Babichenko, Phys. Lett. B 648 (2007) 254.

[21] D. Bernard and A. Leclair, Phys. Lett. B247 (1990) 309.

[22] M. Bershadsky, S. Zhukov and A. Vaintrob, Nucl. Phys. B559 (1999) 205 arXiv:hep-th/9902180.

[23] N. Berkovits, C. Vafa and E. Witten, JHEP 9903 (1999) 018.

[24] D. Bernard, Phys. Lett. B279 (1992) 78.

[25] Al. B. Zamolodchikov, Nucl. Phys. B366 (1991) 122.

[26] C. Candu and H. Saleur, Nucl. Phys. B808 (2009) 487.

[27] C. Candu and H. Saleur, Nucl. Phys. B808 (2009) 441.

[28] V. Mitev, T. Quella and V. Schomerus, JHEP 0811 (2008) 086.

[29] This statement was communicated to us by H. Saleur and by A. Ludwig.

[30] C. Candu, V. Mitev, T. Quella, H. Saleur and V. Schomerus, JHEP 1002 (2010) 015. 